\title{
Actions of Bisnucleophiles on (E)-3-[3-(2-Hydroxyaryl)-3-oxoprop- 1-en-1-yl]chromones: Versatile Transformations into Oxygen- and Nitrogen-Containing Heterocycles
}

\author{
Ridha Hassainea, ${ }^{\mathrm{a}, \mathrm{b}}$ \\ Oualid Talhi ${ }^{* b, c}$ \\ Nadia Taibi ${ }^{b}$ \\ Filipe A Almeida Paz ${ }^{\mathrm{d}}$ \\ Okkacha Bensaid*a \\ Khaldoun Bacharib \\ Artur M. S. Silva*c \\ a Laboratoire de Substances Naturelles et Bio-Actives, \\ Département de Chimie, Faculté des Sciences, Université \\ de Abou Bakr Belkaid Tlemcen, BP 119, 13000, Tlemcen, \\ Algeria \\ ok_bensaid@yahoo.com \\ ${ }^{b}$ Centre de Recherche Scientifique et Technique en \\ Analyses Physico-Chimiques CRAPC, BP 384, Bou-Ismail, \\ 42004, Tipaza, Algeria \\ ' QOPNA, Department of Chemistry, University of Aveiro, \\ 3810-193 Aveiro, Portugal \\ oualid.talhi@ua.pt \\ artur.silva@ua.pt \\ ${ }^{d}$ CICECO - Aveiro Institute of Materials, Department of \\ Chemistry, University of Aveiro, 3810-193 Aveiro, \\ Portugal
}

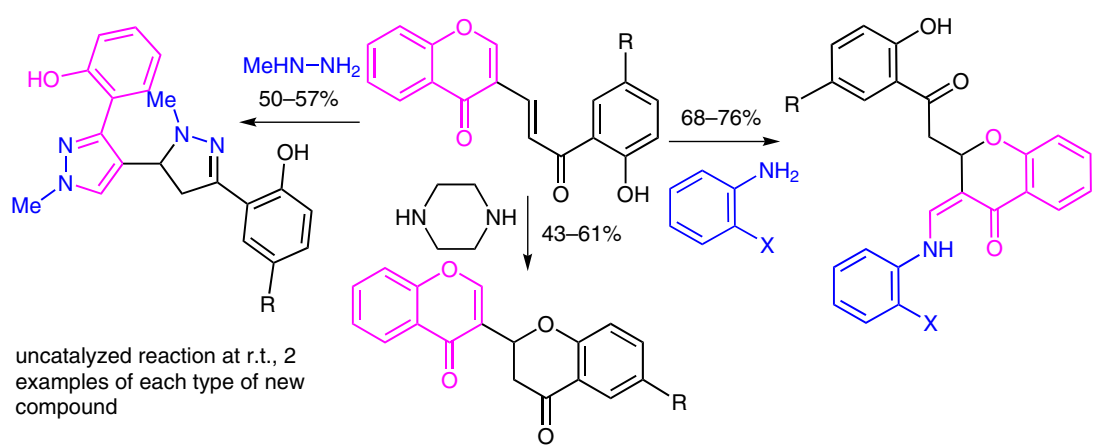

Received: 11.08.2015

Accepted after revision: 04.10.2015

Published online: 05.11.2015

DOI: 10.1055/s-0035-1560829; Art ID: st-2015-d0631-I

Abstract The transformations of (E)-3-[3-(2-hydroxyaryl)-3-oxoprop1-en-1-yl]chromones in the presence of methylhydrazine and aromatic bisnucleophiles are described. The reactions generally lead to chromone ring transformation via pyrone ring-opening and heterocyclization to give novel diazoles and (Z)-3-aminomethylenechromanones, respectively. Piperazine catalyzes chromanone ring closure of the starting substrate to afford chromone-chromanone dyads.

Key words chromones, $\alpha, \beta$-unsaturated carbonyl systems, Michael additions, bisnucleophiles, $\mathrm{X}$-ray diffraction, heterocycles, 2D NMR

Chromones and chromanones (2,3-dihydrochromones) are generally recognized as medicinally active heterocycles. ${ }^{1-3}$ Hence, the important biological properties of these compounds explain their use in many synthetic scaffolds. ${ }^{4}$ Indeed, these types of compounds have long been employed in cancer therapy and anti-inflammatory drug design, especially the flavones. ${ }^{5}$ In addition to their bioactivity, chromones are used as synthetic precursors due to their ability to undergo versatile ring transformations that utilize the intracyclic $\alpha, \beta$-unsaturated carbonyl system of the pyrone ring, leading to enaminochromanones, ${ }^{6}$ aurones $^{7}$ and polyheterocyclic compounds. ${ }^{8}$
In an effort to develop novel biologically active chromanones following new synthetic methods, ${ }^{6,9}$ a considerable amount of work was focused on chromone ring modifications and transformations involving conformational rearrangements ${ }^{10}$ and various cyclizations through hetero-Michael additions. ${ }^{11 a, b}$ The large majority of reactions are nitrogen and oxygen nucleophilic additions promoting ring opening of the pyrone leading to a large diversity of heterocycles. ${ }^{5-11}$ A good example concerns 2-( $N$-methylanilino)-3formylchromones which have demonstrated broad synthetic applications, especially when incorporating a chromone moiety. A variety of heterocyclized chromones, novel macrocycles, and tetradentate ligands can be prepared upon reactions with bisnucleophiles, such as hydrazines and phenylenediamines, via substitution of the $N$-methylanilino moiety and/or condensations with the 3 -formyl group. ${ }^{12}$ Other studies have involved the reaction of 3-formylchromones with active methylene compounds to access different heterocyclic systems, mainly via pyrone ring-opening upon nucleophilic attack ${ }^{13}$ with thiosemicarbazine. Further chelation with nickel(II) yields cytotoxic and DNA-binding agents, ${ }^{14}$ or with diazoles under microwave-assisted conditions to prepare a series of chromone-diazole dyads. ${ }^{15}$

We envisaged that (E)-3-[3-(2-hydroxyaryl)-3-oxoprop1-en-1-yl]chromones 1a,b might undergo further transformations on reactions with bisnucleophiles at their different electrophilic centers, namely the intracyclic $\alpha, \beta$-unsaturat- 
ed carbonyl system (the pyrone ring) along with the exocyclic 3-oxoprop-1-enyl moiety. In this study, methylhydrazine and aromatic-derived bisnucleophiles exhibited different reactivity toward chromones $\mathbf{1 a}, \mathbf{b}$ leading to diazoles 2a,b and 3-aminomethylenechromanones 3aa,ab, respectively (Scheme 1). Moderate to good yields (43-76\%) were achieved overnight at room temperature using tetrahydrofuran as the solvent. Monitoring the reaction progress by thin-layer chromatography showed that the best results were obtained after stirring the reaction for 20 hours at room temperature. Longer reaction times and higher temperatures led to the formation of several other minor products.<smiles>[R]c1ccc(O)c(C(=O)C2Oc3ccccc3C(=O)/C2=C\Nc2ccccc2[Y])c1</smiles>

Scheme 1 Synthesis of diazoles 2a,b, 3-aminomethylenechromanones $\mathbf{3 a a}, \mathbf{a b}$, and chromone-chromanone dyads $\mathbf{4 a}, \mathbf{b}^{\mathbf{1 8}}$

The two transformations of interest involve a domino sequence of nucleophilic attack at the chromone C-2 with pyrone ring opening and pyrazole ring closure, ${ }^{16}$ together with a 1,4-aza-Michael addition to the exocyclic 3-oxoprop-1-enyl moiety followed by formation a 2-pyrazoline ring, ${ }^{17}$ and subsequent nucleophilic attack at the chromone $\mathrm{C}-2$ with pyrone ring opening followed by chromanone ring closure (Scheme 2$)^{5}$

It is worth noting that C-2 of chromones $\mathbf{1}$ is highly reactive toward nucleophiles due to the presence of the oxygen atom at position 1 and the mesomeric electron-withdrawing effects caused by the two carbonyl groups. The
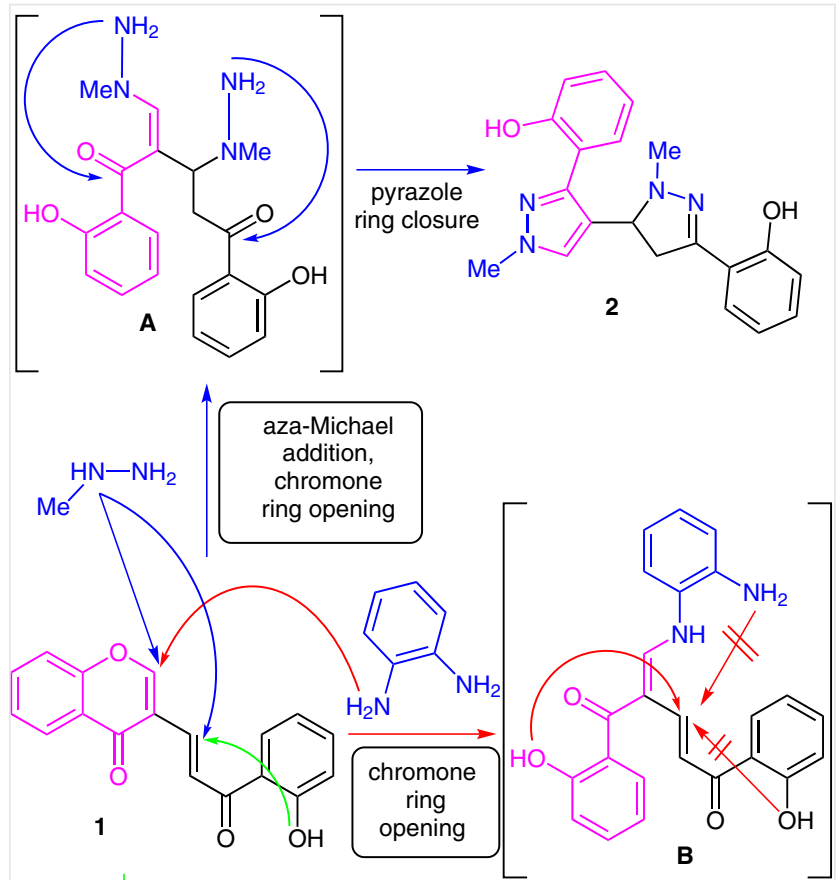<smiles>O=C1CC(c2coc3ccccc3c2=O)Oc2ccccc21</smiles>

\section{$\checkmark$}<smiles>O=Cc1ccccc1O</smiles>

3

Scheme 2 Probable mechanistic pathways for the formation of diazoles 2, 3-aminomethylenechromanones $\mathbf{3}$ and chromone-chromanone dyads 4

presence of a second electrophilic center at $\mathrm{C}-1^{\prime}$ on the 3[(2-hydroxyaryl)-3-oxoprop-1-en-1-yl] moiety of $\mathbf{1}$, even though relatively less electrophilic, allows its participation in a consecutive (or simultaneous) attack of a second molecule of methylhydrazine to form the intermediate $\mathbf{A}$. These events are followed by heterocyclization to afford the diazoles 2 (Scheme 2).

The reactions of aromatic bisnucleophiles, such as phenylenediamine and 2-aminophenol, with chromones $\mathbf{1}$ are expected to proceed through a similar mechanistic pathway, thus favoring the initial nucleophilic attack of nitrogen on the chromone $\mathrm{C}-2$ of the pyrone ring affording intermediate $\mathbf{B}$. Due to the weaker nucleophilic character and bulky nature of these bisnucleophiles, the heterocyclization and/or attack of a second molecule on the electrophilic C -1 ' center of 3-[(2-hydroxyphenyl)-3-oxoprop-1-enyl] moiety 
is not possible. Nevertheless, intermediate $\mathbf{B}$ was able to undergo regioselective intramolecular heterocyclization via an oxa-Michael addition using the hydroxy group of the 2-hydroxyphenyl side chain (resulting from the ring-opening of the pyrone) to yield the corresponding 3-aminomethylenechromanones $\mathbf{3}$ (Scheme 2).

Piperazine, taken as an example of a secondary cyclic diamine, catalyzed the chromanone ring closure of substrates 1 giving rise to the novel chromone-chromanone dyads 4 (Schemes 1 and 2). ${ }^{18}$

2D NMR spectroscopic analysis and single-crystal X-ray diffraction studies were employed to reveal the exact spatial description of structures 2-4. Distinguishing between the 2-(3,4-dihydro-1H-pyrazol-3-yl)phenol and 2-(1H-pyrazol-3-yl)phenol moieties of the diazoles $\mathbf{2 a , b}$ was rather straightforward. ${ }^{19,20}{ }^{1} \mathrm{H}$ NMR spectroscopic analysis clearly showed the AMX spin-system resulting from the asymmetric carbon $\mathrm{C}-3\left(\delta_{\mathrm{C}} 63.8-63.9\right)$ with three doublets of doublets at $\delta_{\mathrm{H}} 2.97-2.98(\mathrm{H}-4[\mathrm{~A}]), 3.65-3.69(\mathrm{H}-4[\mathrm{M}])$, and $4.40-4.42(\mathrm{H}-3[\mathrm{X}])$. The vinylic proton $\mathrm{H}-5^{\prime}$ of the $1 \mathrm{H}$-pyrazole was assigned to a singlet at $\delta_{H} 7.61$. Nevertheless, only analysis of the HMBC connectivities permitted the assignment of the imino carbons at $\delta_{\mathrm{C} 5=\mathrm{N}} 152.9-153.1$ for the 3,4dihydro-1H-pyrazole, and at $\delta_{\mathrm{C}^{\prime}=\mathrm{N}} 148.0-147.9$ for the $1 H$ pyrazole, as both are involved in intramolecular hydrogen bonding with phenolic hydroxy groups $\left(\delta_{2^{\prime \prime}-\mathrm{OH}} 10.56-10.73\right.$ and $\left.\delta_{2 "-\mathrm{OH}} 10.56-10.97\right)$ as was clearly observed from the crystallographic studies (vide infra). The latter proton signals could also be differentiated from their HMBC crosspeak correlations as depicted in Figure 1.

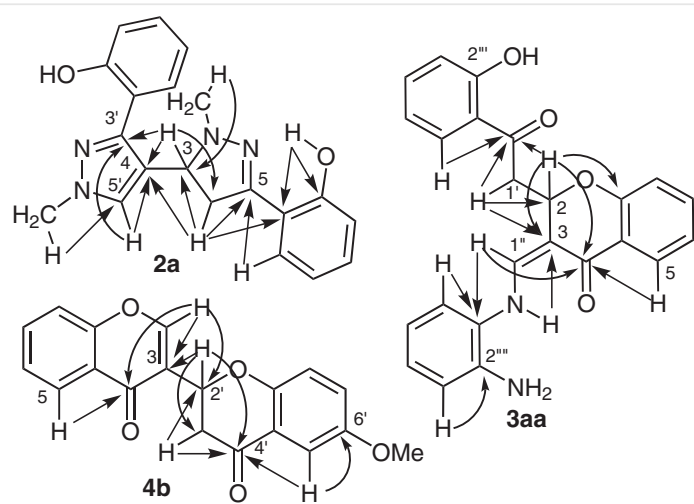

Figure 1 Important HMBC correlations observed in the spectra of compounds $\mathbf{2 a}$, 3 aa and $\mathbf{4 b}$

The 3-aminomethylenechromanones 3aa,ab ${ }^{21,22}$ displayed an ABX spin-system in their ${ }^{1} \mathrm{H}$ NMR spectra $\left(\delta_{\mathrm{H}}\right.$ 3.38-3.43 and 3.69-3.71 for $H-1^{\prime}[A B]$, and 5.73-5.74 for $\mathrm{H}-2[\mathrm{X}]$ ) attributed to the 2-(2-hydroxyaryl)-2-oxoethyl side chain. It was found that the amino protons 1 "-NH appeared as a doublet at $\delta_{1 "-\mathrm{NH}} 11.80-11.99$ due to coupling with the vinylic proton $\mathrm{H}-1$ " $\left(\mathrm{J}_{\mathrm{H} 1 "-1{ }^{\prime N H}}=12.0 \mathrm{~Hz}\right)$ which appear under a multiplet of the aromatic protons $\left(\delta_{\mathrm{H} 1}^{\prime \prime} 7.35-7.57\right)$. The
HMBC connectivities allowed the unequivocal assignment of all the non-protonated carbons (Figure 1). For example, $\mathrm{H}-2[\mathrm{X}]$ showed correlations with the neighboring carbons C-4 $\left(\delta_{\mathrm{C}} 181.4-181.6\right), \mathrm{C}-2^{\prime}\left(\delta_{\mathrm{C}} 203.2-203.3\right), \mathrm{C}-3\left(\delta_{\mathrm{C}} 103.8-\right.$ 103.9) and $C-9\left(\delta_{C} 157.3-157.5\right)$ of the chromanone ring.

The structures of the novel chromone-chromanone dyads $\mathbf{4 a}, \mathbf{b}^{23,24}$ were established unequivocally on the basis of ${ }^{1} \mathrm{H}$ NMR spectroscopic analysis, showing an ABX spin-system for the intracyclic aliphatic protons $H-3$ ' $[A B]$ and $\mathrm{H}-2^{\prime}[\mathrm{X}]$ resulting from the presence of the asymmetric carbon at $C-2^{\prime}\left(\delta_{\mathrm{C}} 73.0-73.1\right)$ of the chromanone unit. Carbonyls $C-4^{\prime}\left(\delta_{C} 191.3-191.5\right)$ and $C-4\left(\delta_{C} 175.7\right)$ could be distinguished using HMBC cross-peak correlations with $\mathrm{H}-5^{\prime}$ and $\mathrm{H}-5$, respectively, as shown in Figure 1.

Single-crystal X-ray diffraction studies provided additional insight on the 3D structures of the chelating hexacyclic iminenol and enaminone systems in diazoles 2 and 3aminomethylenechromanones 3 , respectively.

Crystals of $\mathbf{2} \mathbf{a}^{25}$ were isolated by slow evaporation at $6{ }^{\circ} \mathrm{C}$ from a solution of a $1: 1$ mixture of hexane-dichloromethane. The crystallographic structural features of the diazole 2a were in good agreement with those derived from the 2D NMR studies (Figure 2, top). The 3,4-dihydro-1Hpyrazole group was almost co-planar with the attached phenol group, with the two rings subtending a dihedral angle of $5.18(7)^{\circ}$. On the other hand, the $1 H$-pyrazole group subtended a prominent $25.66(7)^{\circ}$ angle with its corresponding phenol ring. Nevertheless, as depicted in Figure 2, both groups were engaged in $\mathrm{O}-\mathrm{H}$... N intramolecular hydrogen bonds. It is worth emphasizing that the bulky $(3,4-$ dihydro-1H-pyrazol-3-yl)phenol and (1H-pyrazol-3$\mathrm{yl})$ phenol groups of the molecular unit of compound $\mathbf{2 a}$ were located in different planes subtending a dihedral angle of $68.10(4)^{\circ}$.

Crystals of 3-aminomethylenechromanone $\mathbf{3 a a}^{26}$ were obtained from a mixture of hexane-dichloromethane $(1: 1)$ by slow evaporation at $6{ }^{\circ} \mathrm{C}$. Crystallographic studies clearly showed that the chromanone and the phenylenediamine groups were almost coplanar, with their average planes subtending a dihedral angle of only $5.83(5)^{\circ}$. In addition, the intramolecular hydrogen bonding interaction involving these two moieties further promoted the typical Z-configuration, ultimately delineating a hexacyclic enaminone ring (Figure 2, middle). Attached to the C-2 asymmetric center, the 2-(2-hydroxyaryl)-2-oxoethyl side chain subtended a dihedral angle of $31.27(4)^{\circ}$ with that formed by the conjugation of both the chromanone and the phenylenediamine groups.

A similar recrystallization procedure [hexane-dichloromethane $(1: 1)$ at $6{ }^{\circ} \mathrm{C}$ ) allowed the isolation of good quality crystals of the novel chromone-chromanone dyad $\mathbf{4 b} .^{27}$ Crystallographic studies showed that the chromone and 


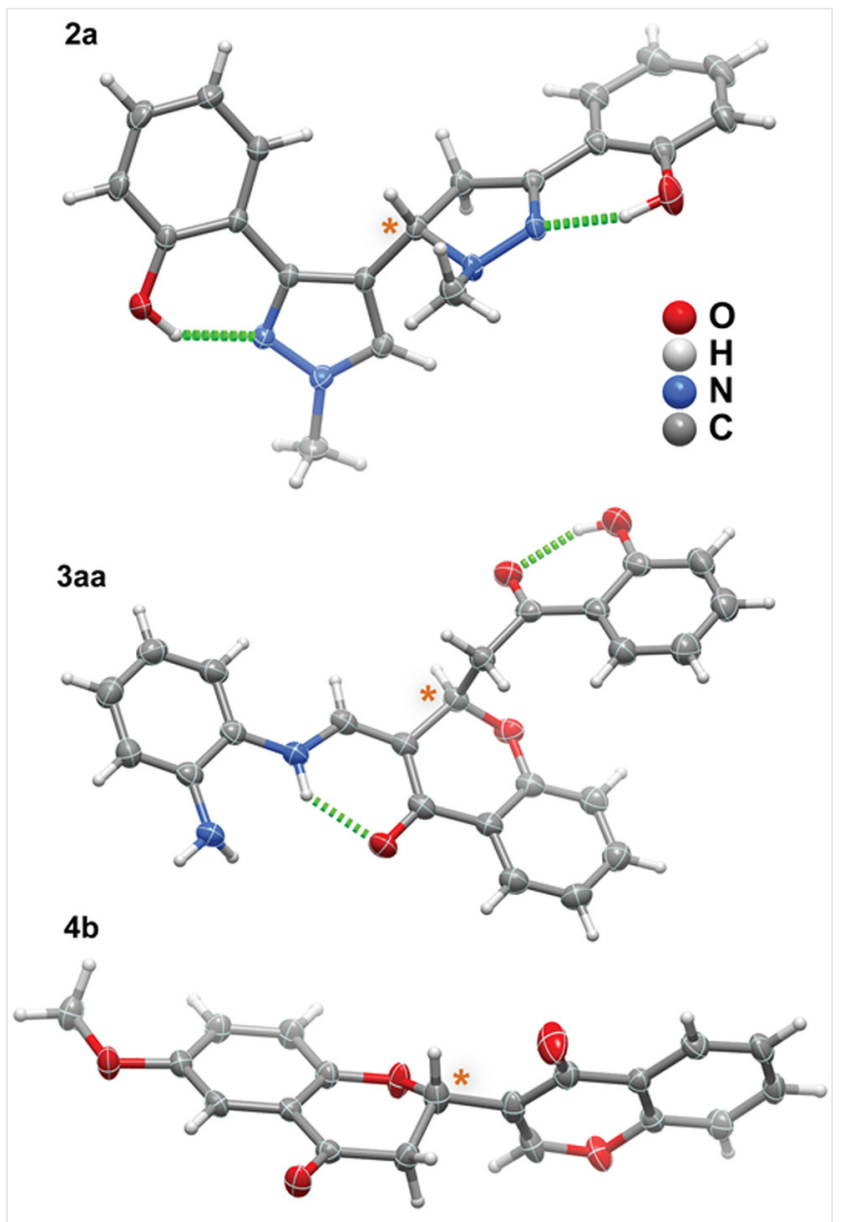

Figure 2 Schematic representations of the molecular units present in compounds 2a, 3aa and $\mathbf{4 b}$. Asymmetric centers are indicated by an asterisk and intramolecular hydrogen bonding interactions as dashed green lines. Non-hydrogen atoms are represented as thermal ellipsoids drawn at the $50 \%$ probability level and hydrogen atoms as small spheres with arbitrary radii.

chromanone average planes, joined together by the asymmetric carbon atom, subtended an average dihedral angle of $72.18(7)^{\circ}$ (Figure 2, bottom).

In conclusion, (E)-3-[3-(2-hydroxyaryl)-3-oxoprop-1en-1-yl]chromones are very reactive substrates toward bisnucleophiles, due to their different electron-deficient centers. The diverse nature of their properties is notably observed from the activated push-pull alkene systems $(\alpha, \beta-$ unsaturated carbonyl systems), providing the ability to undergo nucleophilic additions, followed by pyrone ring opening, ultimately producing versatile intermediates ready for heterocyclizations, thereby allowing the preparation of diazoles $\mathbf{2 a , b},(Z)$-3-aminomethylenechromanones 3aa,ab and chromone-chromanone dyads 4 .

\section{Acknowledgment}

We are grateful to the University of Aveiro and FCT/MEC for financial support to the QOPNA research project (FCT UID/QUI/00062/2013), CICECO-Aveiro Institute of Materials (FCT UID/CTM/50011/2013) through national funds and, where applicable, co-financed by FEDER, within the PT2020 Partnership Agreement, and to the Portuguese National NMR Network (RNRMN). We would like to thank the General Directorate for Scientific Research and Technological Development (DGRSDT) of Algeria for financial support to O.T. Thanks are due to the project 'New Strategies Applied to Neuropathological Disorders' (CENTRO-07-ST24-FEDER-002034), co-funded by QREN, 'Mais Centro-Programa Operacional Regional do Centro' and EU, FEDER for postdoctoral funding to O.T. We also wish to thank CICECO for specific funding toward the purchase of a single-crystal X-ray diffractometer.

\section{Supporting Information}

Supporting information for this article is available online at http://dx.doi.org/10.1055/s-0035-1560829.

\section{References and Notes}

(1) (a) Ellis, G. P. Chromenes, Chromanones and Chromones; Wiley: New York, 1977. (b) Andersen, Ø. M.; Markham, K. R. Flavonoids: Chemistry, Biochemistry and Applications; Taylor \& Francis: Boca Raton, 2006. (c) Kabbe, H. J.; Widdig, A. Angew. Chem., Int. Ed. Engl. 1982, 21, 247.

(2) (a) Saengchantara, S. T.; Wallace, T. W. Nat. Prod. Rep. 1986, 3, 465. (b) Cottiglia, F.; Dhanapal, B.; Sticher, O.; Heilmann, J. J. Nat. Prod. 2004, 67, 537. (c) Carpenter, R. D.; DeBerdt, P. B.; Holden, J. B.; Milinkevich, K. A.; Min, T.; Willenbring, D.; Fettinger, J. C.; Tantillo, D. J.; Kurth, M. J. J. Comb. Chem. 2008, 10, 225. (d) Carpenter, R. D.; Fettinger, J. C.; Lam, K. S.; Kurth, M. J. Angew. Chem. Int. Ed. 2008, 47, 6407.

(3) (a) Chandrasekhar, S.; Vijeender, K.; Reddy, K. V. Tetrahedron Lett. 2005, 46, 6991. (b) Graves, C. R.; Zeng, B. S.; Nguyen, S. T. J. Am. Chem. Soc. 2006, 128, 12596. (c) Enders, D.; Breuer, K.; Runsink, J.; Teles, J. H. Helv. Chim. Acta 1996, 79, 1899. (d) McKervey, M. A.; Ye, T. J. Chem. Soc., Chem. Commun. 1992, 823. (e) Biddle, M. M.; Lin, M.; Scheidt, K. A. J. Am. Chem. Soc. 2007, 129, 3830. (f) Butler, J. D.; Conrad, W. E.; Lodewyk, M. W.; Fettinger, J. C.; Tantillo, D. J.; Kurth, M. J. Org Lett. 2010, 12, 3410.

(4) (a) Takikawa, H.; Suzuki, K. Org. Lett. 2007, 9, 2713. (b) Majewski, M.; Irvine, N. M.; Bantle, G. W. J. Org. Chem. 1994, 59, 6697. (c) Lipinski, C. A.; Aldinger, C. E.; Beyer, T. A.; Bordner, J.; Bussolotti, D. F.; Inskeep, P. B.; Siegel, T. W. J. Med. Chem. 1992, 35, 2169.

(5) (a) Talhi, O.; Silva, A. M. S. Curr. Org. Chem. 2012, 16, 859. (b) Talhi, O.; Silva, A. M. S. Curr. Org. Chem. 2013, 17, 1067. (c) Talhi, O.; Pinto, D. C. G. A.; Silva, A. M. S. The French Paradox at Tea Time: From Flavonoids Toward Synthetic Stilbene-Based Drugs, In Recent Advances in Redox Active Plant and Microbial Products; Jacob, C.; Kirsch, G.; Slusarenko, A. J.; Winyard, P. G.; Burkholz, T., Eds.; Springer: Dordrecht, 2014, 149-189.

(6) (a) Pintiala, C.; Lawson, A. M.; Comesse, S.; Daïch, A. Tetrahedron Lett. 2013, 54, 2853. (b) Wu, C.; Liu, Y.; Zeng, H.; Liu, L.; Wang, D.; Chen, Y. Org. Biomol. Chem. 2011, 9, 253.

(7) Huang, W.; Liu, M.-Z.; Li, Y.; Tan, Y.; Yang, G.-F. Bioorg. Med. Chem. 2007, 15, 5191. 
(8) Lévai, A.; Simon, A.; Jenei, A.; Kálmán, G.; Jekő, J.; Tóth, G. ARKIVOC 2009, (xii), 161.

(9) (a) Fridén-Saxin, M.; Pemberton, N.; Andersson, K. S.; Dyrager, C.; Friberg, A.; Grøtli, M.; Luthman, K. J. Org. Chem. 2009, 74, 2755. (b) Wu, C.; Zeng, H.; Liu, L.; Wang, D.; Chen, Y. Tetrahedron 2011, 67, 1231.

(10) Budzisz, E.; Pastuszko, S. Tetrahedron 1999, 55, 4815.

(11) (a) Vyacheslav, Y. S.; Vladimir, S. M.; Mikhail, I. K. Tetrahedron 2008, 64, 7877. (b) Santos, C. M. M.; Silva, A. M. S.; Jekő, J.; Lévai, A. ARKIVOC 2012, (v), 265. (c) Mkrtchyan, S.; Iaroshenko, V. O.; Dudkin, S.; Gevorgyan, A.; Vilches-Herrera, M.; Ghazaryan, G.; Volochnyuk, D. M.; Ostrovskyi, D.; Ahmed, Z.; Villinger, A.; Sosnovskikh, V. Y.; Langer, P. Org. Biomol. Chem. 2010, 8, 5280.

(12) Singh, G.; Singh, L.; Ishar, M. P. S. Tetrahedron 2002, 58, 7883.

(13) Gašparová, R.; Lácová, M. Molecules 2005, 10, 937.

(14) Wang, B.-D.; Yang, Z.-Y.; Lu, M.-H.; Hai, J.; Wang, Q.; Chen, Z.-N. J. Organomet. Chem. 2009, 694, 4069.

(15) Lacova, M.; Gasparova, R.; Loos, D.; Liptay, T.; Pronayova, N. Molecules 2000, 5, 167.

(16) (a) Pinto, D. C. G. A.; Silva, A. M. S.; Cavaleiro, J. A. S.; FocesFoces, C.; Llamas-Sainz, A.; Jagerovic, N.; Elguero, J. Tetrahedron 1999, 55, 10187. (b) Pinto, D. C. G. A.; Silva, A. M. S.; Cavaleiro, J. A. S. J. Heterocycl. Chem. 2000, 37, 1629. (c) Lévai, A.; Silva, A. M. S.; Cavaleiro, J. A. S.; Alkorta, I.; Elguero, J.; Jekő, J. Eur. J. Org. Chem. 2006, 2825. (d) Silva, V. L. M.; Silva, A. M. S.; Pinto, D. C. G. A.; Cavaleiro, J. A. S.; Elguero, J. Tetrahedron Lett. 2007, 48, 3859.

(17) (a) Pinto, D. C. G. A.; Silva, A. M. S.; Lévai, A.; Cavaleiro, J. A. S.; Patonay, T.; Elguero, J. Eur.J. Org. Chem. 2000, 2593. (b) Lévai, A.; Patonay, T.; Silva, A. M. S.; Pinto, D. C. G. A.; Cavaleiro, J. A. S. J. Heterocycl. Chem. 2002, 39, 751. (c) Pinto, D. C. G. A.; Silva, A. M. S.; Lévai, A.; Cavaleiro, J. A. S.; Elguero, J. Eur. J. Org. Chem. 2003, 747. (d) Lévai, A.; Silva, A. M. S.; Pinto, D. C. G. A.; Cavaleiro, J. A. S.; Alkorta, I.; Elguero, J.; Jekő, J. Eur. J. Org. Chem. 2004, 4672.

(18) Transformations of (E)-3-[3-(2-Hydroxyaryl)-3-oxoprop-1en-1-yl]chromones 1a,b; General procedure

Methylhydrazine, phenylenediamine, 2-aminophenol or piperazine $(2 \mathrm{mmol})$ was added to a stirred solution of $(E)-3-[3-(2-$ hydroxyaryl)-3-oxoprop-1-en-1-yl]chromone $\mathbf{1 a , b}$ (1 mmol) in THF $(20 \mathrm{~mL})$ at r.t., and the resulting mixture was allowed to stir overnight. After removal of the solvent under reduced pressure, the obtained resinous material was subjected to column chromatography. The product was recrystallized from a mixture of hexane-dichloromethane $(1: 1)$ by slow evaporation of the solvent at $6{ }^{\circ} \mathrm{C}$ to afford 2a,b, 3aa,ab or $\mathbf{4 a , b}$. Good-quality single crystals suitable for X-ray analysis were only obtained for products 2a, 3aa and $\mathbf{4 a , b}$.

(19) 2,2'-(1',2-Dimethyl-3,4-dihydro-1'H,2H-[3,4'-bispyrazole]3',5-diyl)diphenol (2a)

Yield: $0.20 \mathrm{~g}(57 \%) ; M_{\mathrm{r}}=348.41 \mathrm{~g} / \mathrm{mol}$; colorless crystals; $\mathrm{mp}$ 205-206 ${ }^{\circ} \mathrm{C}$. ${ }^{1} \mathrm{H}$ NMR $\left(300 \mathrm{MHz}, \mathrm{CDCl}_{3}\right): \delta=2.88(\mathrm{~s}, 3 \mathrm{H}, 1-\mathrm{N}-$ $\mathrm{CH}_{3}$ ), 2.98 (dd, $J=18.0,14.0 \mathrm{~Hz}, 1 \mathrm{H}, \mathrm{H}-4[\mathrm{~A}]$ ), 3.69 (dd, $J=18.0$, $10.0 \mathrm{~Hz}, 1 \mathrm{H}, \mathrm{H}-4[\mathrm{M}]), 3.95$ (s, $\left.3 \mathrm{H}, 1^{\prime}-\mathrm{N}-\mathrm{CH}_{3}\right), 4.40$ (dd, $J=14.0$, $10.0 \mathrm{~Hz}, 1 \mathrm{H}, \mathrm{H}-3[\mathrm{X}]), 6.83-6.94$ (m, $\left.2 \mathrm{H}, \mathrm{H}-5^{\prime \prime}, \mathrm{H}-5^{\prime \prime \prime}\right), 6.97-7.14$ (m, 3 H, H-3", H-3"', H-6"), 7.20-7.28 (m, 2 H, H-4", H-4"'), 7.47 (dd, $\left.J=7.8,1.6 \mathrm{~Hz}, 1 \mathrm{H}, \mathrm{H}-6^{\prime \prime \prime}\right), 7.61$ (s, $\left.1 \mathrm{H}, \mathrm{H}-5^{\prime}\right), 10.73$ (s, $1 \mathrm{H}$, 2"'-OH), 10.97 (s, $1 \mathrm{H}, 2$ "-OH). ${ }^{13} \mathrm{C}$ NMR $\left(75 \mathrm{MHz}, \mathrm{CDCl}_{3}\right)$ : $\delta=39.1\left(1^{\prime}-\mathrm{N}-\mathrm{CH}_{3}\right), 42.1$ and $42.2\left(1-\mathrm{N}-\mathrm{CH}_{3}\right.$ and $\left.\mathrm{C}-4\right), 63.8(\mathrm{C}-3)$, 116.1 (C-1"), 116.5 (C-3"), 117.2 (C-1"'), 117.3 (C-3"'), 118.3 (C4'), 119.1 (C-5"), 119.2 (C-5"'), 127.2 (C-6"), 127.3 (C-6"'), 129.1 (C-4"), 130.4 (C-4'"'), 130.5 (C-5'), 147.9 (C-3'), 153.1 (C-5), 155.7 (C-2"'), 157.6 (C-2"). HRMS (ESI): $m / z[\mathrm{M}+\mathrm{H}]^{+}$calcd for $\mathrm{C}_{20} \mathrm{H}_{21} \mathrm{~N}_{4} \mathrm{O}_{2}$ : 349.1665; found: 349.1668 .
(20) 2-[3'-(2-Hydroxyphenyl)-1',2-dimethyl-3,4-dihydro-1'H,2H[3,4'-bispyrazol]-5-yl]-4-methoxyphenol (2b)

Yield: $0.19 \mathrm{~g}(50 \%) ; M_{\mathrm{r}}=378.43 \mathrm{~g} / \mathrm{mol}$; white solid; $\mathrm{mp} 182-$ $183^{\circ} \mathrm{C} .{ }^{1} \mathrm{H}$ NMR $\left(300 \mathrm{MHz}, \mathrm{CDCl}_{3}\right): \delta=2.87\left(\mathrm{~s}, 3 \mathrm{H}, 1-\mathrm{N}-\mathrm{CH}_{3}\right)$, 2.97 (dd, $J=18.0,14.0 \mathrm{~Hz}, 1 \mathrm{H}, \mathrm{H}-4[\mathrm{~A}]$ ), 3.65 (dd, $J=18.0,10.0$ $\mathrm{Hz}, 1 \mathrm{H}, \mathrm{H}-4[\mathrm{M}]), 3.74$ (s, $3 \mathrm{H}, 5^{\prime \prime}-\mathrm{OCH}_{3}$ ), 3.95 (s, $3 \mathrm{H}, 1^{\prime}-\mathrm{N}-\mathrm{CH}_{3}$ ), 4.42 (dd, $J=14.0,10.0 \mathrm{~Hz}, 1 \mathrm{H}, \mathrm{H}-3[\mathrm{X}]), 6.62$ (d, J=2.9 Hz, $1 \mathrm{H}$, H-6"), 6.81-6.95 (m, 3 H, H-4", H-3", H-5"'), 7.06 (dd, J = 8.2, 1.2 $\mathrm{Hz}, 1 \mathrm{H}, \mathrm{H}-3^{\prime \prime \prime}$ ), 7.20-7.27 (m, $1 \mathrm{H}, \mathrm{H}-4^{\prime \prime \prime}$ ), 7.47 (dd, $J=7.8,1.6$ $\left.\mathrm{Hz}, 1 \mathrm{H}, \mathrm{H}-6^{\prime \prime \prime}\right), 7.61$ (s, $\left.1 \mathrm{H}, \mathrm{H}-5^{\prime}\right), 10.56$ (br s, $2 \mathrm{H}, 2^{\prime \prime \prime}-\mathrm{OH}$ and $2 "-\mathrm{OH}) .{ }^{13} \mathrm{C}$ NMR $\left(75 \mathrm{MHz}, \mathrm{CDCl}_{3}\right): \delta=39.2\left(1^{\prime}-\mathrm{N}-\mathrm{CH}_{3}\right), 42.1$ and $42.3\left(1-\mathrm{N}-\mathrm{CH}_{3}\right.$ and $\left.\mathrm{C}-4\right), 55.9\left(5^{\prime \prime}-\mathrm{O}-\mathrm{CH}_{3}\right), 63.9(\mathrm{C}-3), 112.2(\mathrm{C}-$ 6"), 116.3 (C-4"), 117.1 (C-3"), 117.3 (C-1"'), 117.4 (C-3"'), 118.4 (C-5"'), 119.4 (C-4'), 127.3 (C-6"'), 129.2 (C-4'"'), 130.7 (C-5'), 148.0 (C-3'), 151.9 (C-5"), 152.3 (C-1"), 152.9 (C-5), 155.8 and 156.0 (C-2" and C-2"'). HRMS (ESI): $m / z[\mathrm{M}+\mathrm{Na}]^{+}$calcd for $\mathrm{C}_{21} \mathrm{H}_{22} \mathrm{~N}_{4} \mathrm{O}_{3} \mathrm{Na}$ : 401.1590; found: 401.1581 .

(21) (Z)-3-\{[(2-Aminophenyl)amino]methylene\}-2-[2-(2hydroxyphenyl)-2-oxoethyl]chroman-4-one (3aa)

Yield: $0.27 \mathrm{~g}(68 \%), M_{\mathrm{r}}=400.43 \mathrm{~g} / \mathrm{mol}$; orange crystals; $\mathrm{mp} 140-$ $141{ }^{\circ} \mathrm{C} .{ }^{1} \mathrm{H}$ NMR $\left(300 \mathrm{MHz}, \mathrm{CDCl}_{3}\right): \delta=3.43(\mathrm{dd}, J=16.4,7.0 \mathrm{~Hz}$, $\left.1 \mathrm{H}, \mathrm{H}-1^{\prime}[\mathrm{AB}]\right), 3.69$ (dd, $\left.J=16.4,6.2 \mathrm{~Hz}, 1 \mathrm{H}, \mathrm{H}-1^{\prime}[\mathrm{AB}]\right), 3.70$ (s, 2 $\mathrm{H}, 2^{\prime \prime \prime '-} \mathrm{NH}_{2}$ ), 5.73 (dd, J = 7.0, 6.2 Hz, 1 H, H-2[X]), 6.76-6.91 (m, 4 H, H-5"', H-3"', H-4"'", H-6"'"'), 6.93-7.12 (m, 4 H, H-8, H-6, H3"', H-5'"'), 7.38-7.54 (m, 4 H, H-7, H-4"', H-1", H-6"'), 7.97 (dd, $J=7.7,1.3 \mathrm{~Hz}, 1 \mathrm{H}, \mathrm{H}-5), 11.80$ (d, $\left.J=12.0 \mathrm{~Hz}, 1 \mathrm{H}, 1^{\prime \prime}-\mathrm{NH}\right), 12.18$ (s, $1 \mathrm{H}, 2$ "'--OH). ${ }^{13} \mathrm{C}$ NMR (75 MHz, $\mathrm{CDCl}_{3}$ ): $\delta=44.3\left(\mathrm{C}-1^{\prime}\right), 75.3$ (C-2), 103.8 (C-3), 117.4 (C-3"'"'), 117.9 (C-6"'"'), 118.1 (C-4"'"'), 118.6 (C-8), 119.1 (C-5"'), 119.5 (C-1"'), 120.1 (C-3"'), 121.9 (C6), 122.8 (C-10), 125.6 (C-5"'"), 126.5 (C-5), 128.2 (C-1"'"'), 130.1 (C-6"'), 134.6 (C-7), 136.9 (C-4"'), 137.2 (C-2"'"'), 144.5 (C-1"), 157.3 (C-9), 162.6 (C-2'"'), 181.6 (C-4), 203.3 (C-2'). HRMS (ESI): $\mathrm{m} / z[\mathrm{M}+\mathrm{Na}]^{+}$calcd for $\mathrm{C}_{24} \mathrm{H}_{20} \mathrm{~N}_{2} \mathrm{O}_{4} \mathrm{Na}$ : 423.1321; found: 423.1318.

(22) (Z)-2-[2-(2-Hydroxyphenyl)-2-oxoethyl]-3-\{[(2-hydroxyphenyl)amino]methylene \}chroman-4-one (3ab)

Yield: $0.27 \mathrm{~g}(76 \%) ; M_{\mathrm{r}}=401.41 \mathrm{~g} / \mathrm{mol}$; yellow solid; $\mathrm{mp} 140$ $141{ }^{\circ} \mathrm{C} .{ }^{1} \mathrm{H}$ NMR $\left(300 \mathrm{MHz}, \mathrm{CDCl}_{3}\right): \delta=3.38(\mathrm{dd}, J=16.2,6.6 \mathrm{~Hz}$, $\left.1 \mathrm{H}, \mathrm{H}-1^{\prime}[\mathrm{AB}]\right), 3.71$ (dd, $\left.J=16.2,6.6 \mathrm{~Hz}, 1 \mathrm{H}, \mathrm{H}-1^{\prime}[\mathrm{AB}]\right), 5.74(\mathrm{t}$, $J=6.6 \mathrm{~Hz}, 1 \mathrm{H}, \mathrm{H}-2[\mathrm{X}]), 6.75-6.99$ (m, $6 \mathrm{H}, \mathrm{H}-4^{\prime \prime \prime \prime}, \mathrm{H}-6$ '"', H-5'"', H-3"', H-5"', H-8), 7.03-7.17 (m, 2 H, H-6, H-3"'"), 7.35-7.57 (m, 4 H, H-7, H-4"', H-6"', H-1"), 8.01 (dd, J = 7.8, $1.7 \mathrm{~Hz}, 1 \mathrm{H}, \mathrm{H}-5$ ), $11.99\left(\mathrm{~d}, J=12.0 \mathrm{~Hz}, 1 \mathrm{H}, 1^{\prime \prime}-\mathrm{NH}\right), 12.11\left(\mathrm{~s}, 1 \mathrm{H}, 2^{\prime \prime \prime}-\mathrm{OH}\right) .{ }^{13} \mathrm{C}$ NMR $\left(75 \mathrm{MHz}, \mathrm{CDCl}_{3}\right): \delta=44.4\left(\mathrm{C}-1^{\prime}\right), 75.6(\mathrm{C}-2), 103.9(\mathrm{C}-3), 114.6$ (C-3"'"'), 116.2 (C-6"'"'), 118.1 (C-4"'"'), 118.6 (C-8), 119.0 (C-5"'), 119.6 (C-1"'), 121.3 (C-3"'), 121.8 (C-6), 122.9 (C-10), 124.3 (C5'"'), 126.6 (C-5), 128.5 (C-1"'"'), 130.1 (C-6"'), 134.5 (C-7), 136.7 (C-4"'), 141.8 (C-1"'), 145.5 (C-2'"'), 157.5 (C-9), 162.7 (C-2"'), 181.4 (C-4), $203.2\left(\mathrm{C}-2^{\prime}\right)$. HRMS (ESI): $m / z[\mathrm{M}+\mathrm{Na}]^{+}$calcd for $\mathrm{C}_{24} \mathrm{H}_{19} \mathrm{NO}_{5} \mathrm{Na}$ : 424.1161; found: 424.1131 .

(23) 3-(4-0xochroman-2-yl)-4H-chromen-4-one (4a)

Yield: $0.18 \mathrm{~g}(61 \%) ; M_{\mathrm{r}}=292.29 \mathrm{~g} / \mathrm{mol}$; colorless crystals; $\mathrm{mp}$ 195-196 ${ }^{\circ} \mathrm{C} .{ }^{1} \mathrm{H}$ NMR $\left(300 \mathrm{MHz}, \mathrm{CDCl}_{3}\right): \delta=2.89$ (dd, $J=16.9$, $\left.13.0 \mathrm{~Hz}, 1 \mathrm{H}, \mathrm{H}-3^{\prime}[\mathrm{AB}]\right), 3.25$ (dd, $J=16.9,3.0 \mathrm{~Hz}, 1 \mathrm{H}, \mathrm{H}^{\prime} 3^{\prime}[\mathrm{AB}]$ ), 5.72 (ddd, $\left.J=13.0,3.0,1.0 \mathrm{~Hz}, 1 \mathrm{H}, \mathrm{H}-2^{\prime}[\mathrm{X}]\right), 7.02-7.13(\mathrm{~m}, 2 \mathrm{H}$, H-8', H-6'), 7.40-7.57 (m, 3 H, H-6, H-8, H-7'), 7.72 (ddd, $J=8.7$, 7.1, $1.7 \mathrm{~Hz}, 1 \mathrm{H}, \mathrm{H}-7$ ), 7.95 (ddd, $J=8.6,4.9,3.6 \mathrm{~Hz}, 1 \mathrm{H}, \mathrm{H}-5^{\prime}$ ), 8.20-8.28 (m, $1 \mathrm{H}, \mathrm{H}-5), 8.24$ (d, $J=1.0 \mathrm{~Hz}, 1 \mathrm{H}, \mathrm{H}-2) .{ }^{13} \mathrm{C}$ NMR $\left(75 \mathrm{MHz}, \mathrm{CDCl}_{3}\right): \delta=42.8\left(\mathrm{C}-3^{\prime}\right), 73.0\left(\mathrm{C}-2^{\prime}\right), 117.9\left(\mathrm{C}-8^{\prime}\right), 118.2$ (C-8), $121.1\left(\mathrm{C}-10^{\prime}\right), 122.0\left(\mathrm{C}-6^{\prime}\right), 122.9(\mathrm{C}-3), 123.8(\mathrm{C}-10)$, 125.5 (C-6), 125.9 (C-5), 127.3 (C-5'), 134.1 (C-7), 136.1 (C-7'), 
153.3 (C-2), 156.3 (C-9), 161.2 (C-9'), 175.7 (C-4), 191.3 (C-4'). HRMS (ESI): $m / z$ [M $+\mathrm{Na}]^{+}$calcd for $\mathrm{C}_{18} \mathrm{H}_{12} \mathrm{O}_{4} \mathrm{Na}$ : 315.0633; found: 315.0636 .

(24) 3-(6-Methoxy-4-oxochroman-2-yl)-4H-chromen-4-one (4b) Yield: $0.14 \mathrm{~g}(43 \%) ; M_{\mathrm{r}}=322.31 \mathrm{~g} / \mathrm{mol}$; colorless crystals; $\mathrm{mp}$ 195-196 ${ }^{\circ} \mathrm{C} .{ }^{1} \mathrm{H}$ NMR $\left(300 \mathrm{MHz}, \mathrm{CDCl}_{3}\right): \delta=2.85$ (dd, $J=17.0$, $\left.13.1 \mathrm{~Hz}, 1 \mathrm{H}, \mathrm{H}-3^{\prime}[\mathrm{AB}]\right), 3.24$ (dd, J = 17.0, $2.9 \mathrm{~Hz}, 1 \mathrm{H}, \mathrm{H}-3^{\prime}[\mathrm{AB}]$ ), $3.83\left(\mathrm{~s}, 3 \mathrm{H}, 6^{\prime}-\mathrm{OCH}_{3}\right.$ ), 5.67 (ddd, $J=13.1,2.9,1.0 \mathrm{~Hz}, 1 \mathrm{H}, \mathrm{H}-$ $2^{\prime}[\mathrm{X}]$ ), 7.00 (d, $\left.J=9.0 \mathrm{~Hz}, 1 \mathrm{H}, \mathrm{H}-8^{\prime}\right), 7.13$ (dd, $J=9.0,3.2 \mathrm{~Hz}, 1 \mathrm{H}$, H-7'), 7.37 (d, J = 3.1 Hz, 1 H, H-5'), 7.40-7.52 (m, 2 H, H-6, H-8), 7.72 (ddd, $J=8.6,7.2,1.7 \mathrm{~Hz}, 1 \mathrm{H}, \mathrm{H}-7), 8.21-8.28(\mathrm{~m}, 1 \mathrm{H}, \mathrm{H}-5)$, $8.24(\mathrm{~d}, J=1.0 \mathrm{~Hz}, 1 \mathrm{H}, \mathrm{H}-2) .{ }^{13} \mathrm{C} \mathrm{NMR}\left(75 \mathrm{MHz}, \mathrm{CDCl}_{3}\right): \delta=42.7$ $\left(\mathrm{C}-3^{\prime}\right), 55.8\left(6^{\prime}-\mathrm{OCH}_{3}\right) 73.1\left(\mathrm{C}-2^{\prime}\right), 107.6\left(\mathrm{C}-5^{\prime}\right), 118.2(\mathrm{C}-8), 119.2$ (C-8'), $121.0\left(\mathrm{C}-10^{\prime}\right), 123.0(\mathrm{C}-3), 123.8$ (C-10), 125.2 (C-7'), 125.5 (C-6), 125.9 (C-5), 134.0 (C-7), 153.3 (C-2), 154.5 (C-6'), 155.8 (C-9'), 156.3 (C-9), 175.7 (C-4), 191.5 (C-4'). HRMS (ESI): $\mathrm{m} / z[\mathrm{M}+\mathrm{Na}]^{+}$calcd for $\mathrm{C}_{19} \mathrm{H}_{14} \mathrm{O}_{5} \mathrm{Na}$ : 345.0739 ; found: 345.0748 .

(25) Crystal data for compound 2a (CCDC 1404991): $\mathrm{C}_{20} \mathrm{H}_{20} \mathrm{~N}_{4} \mathrm{O}_{2}$, $M=348.40$, triclinic, space group $P_{\overline{1}}, Z=2, a=8.7610(13) \AA$, $b=9.9194(16) \AA, c=11.871(2) \AA, \alpha=66.580(7)^{\circ}, \beta=70.069(7)^{\circ}$, $\gamma=74.993(7)^{\circ}, \quad V=880.8(3) \AA^{3}, \quad \mu(\mathrm{Mo}-\mathrm{K} \alpha)=0.088 \mathrm{~mm}^{-1}$, $D_{\mathrm{c}}=1.314 \mathrm{~g} \mathrm{~cm}^{-3}$, colorless block, crystal size $=$
$0.22 \times 0.16 \times 0.10 \mathrm{~mm}^{3}$. Of a total of 19322 reflections collected, 4753 were independent $\left(R_{\text {int }}=0.0413\right)$. Final $R 1=0.0467$ $[I>2 \sigma(I)]$ and $w R 2=0.1267$ (all data). Data completeness to $\theta=25.24^{\circ}, 99.9 \%$.

(26) Crystal data for compound 3aa (CCDC 1404990): $\mathrm{C}_{24} \mathrm{H}_{20} \mathrm{~N}_{2} \mathrm{O}_{4}$, $M=400.42$, monoclinic, space group $P 2_{1} / n, \quad Z=4$, $a=14.9755(11) \AA, \quad b=4.7510(3) \AA, \quad c=26.6395(16) \AA$, $\beta=93.664(5)^{\circ}, \quad V=1891.5(2) \AA^{3}, \quad \mu($ Mo-K $\alpha)=0.097 \mathrm{~mm}^{-1}$, $D_{\mathrm{c}}=1.406 \mathrm{~g} \mathrm{~cm}^{-3}$, orange plate, crystal size of $0.22 \times 0.12 \times 0.03$ $\mathrm{mm}^{3}$. Of a total of 15482 reflections collected, 4999 were independent $\left(R_{\text {int }}=0.0475\right)$. Final $R 1=0.0505 \quad[I>2 \sigma(I)]$ and $w R 2=0.1233$ (all data). Data completeness to $\theta=25.24^{\circ}, 99.6 \%$.

(27) Crystal data for compound 4b (CCDC 1404989): $\mathrm{C}_{19} \mathrm{H}_{14} \mathrm{O}_{5}$, $M=322.30$, triclinic, space group $P \overline{1}, Z=2, a=8.4108(9) \AA$, $b=8.5434(9) \AA, \quad c=10.6878(11) \AA, \quad \alpha=83.293(3)^{\circ}$, $\beta=72.084(3)^{\circ}, \gamma=82.141(3)^{\circ}, \quad V=721.61(13) \AA^{3}, \quad \mu(\mathrm{Mo}-\mathrm{K} \alpha)=$ $0.108 \mathrm{~mm}^{-1}, D_{\mathrm{c}}=1.483 \mathrm{~g} \mathrm{~cm}^{-3}$, colorless plate, crystal size of $0.16 \times 0.12 \times 0.07 \mathrm{~mm}^{3}$. Of a total of 13854 reflections collected, 2640 were independent $\left(R_{\text {int }}=0.0256\right)$. Final $R 1=0.0553$ $[I>2 \sigma(I)]$ and $w R 2=0.1356$ (all data). Data completeness to $\theta=25.24^{\circ}, 99.6 \%$. 